\title{
Prediction of Currency Volume Issued in Taiwan Using a Hybrid Artificial Neural Network and Multiple Regression Approach
}

\author{
Yuehjen E. Shao \\ Department of Statistics and Information Science, Fu Jen Catholic University, Xinzhuang, New Taipei 24205, Taiwan \\ Correspondence should be addressed to Yuehjen E. Shao; stat1003@mail.fu.edu.tw
}

Received 12 December 2012; Revised 22 February 2013; Accepted 23 February 2013

Academic Editor: Ker-Wei Yu

Copyright (C) 2013 Yuehjen E. Shao. This is an open access article distributed under the Creative Commons Attribution License, which permits unrestricted use, distribution, and reproduction in any medium, provided the original work is properly cited.

\begin{abstract}
Because the volume of currency issued by a country always affects its interest rate, price index, income levels, and many other important macroeconomic variables, the prediction of currency volume issued has attracted considerable attention in recent years. In contrast to the typical single-stage forecast model, this study proposes a hybrid forecasting approach to predict the volume of currency issued in Taiwan. The proposed hybrid models consist of artificial neural network (ANN) and multiple regression (MR) components. The MR component of the hybrid models is established for a selection of fewer explanatory variables, wherein the selected variables are of higher importance. The ANN component is then designed to generate forecasts based on those important explanatory variables. Subsequently, the model is used to analyze a real dataset of Taiwan's currency from 1996 to 2011 and twenty associated explanatory variables. The prediction results reveal that the proposed hybrid scheme exhibits superior forecasting performance for predicting the volume of currency issued in Taiwan.
\end{abstract}

\section{Introduction}

The Central Bank of Taiwan is responsible for planning and producing the currency of the country. The volume of currency issued is primarily determined by the demand of the public. It is associated with the economic growth rate, seasonal factors, and the development of noncash payments. Because the issue of currency is very important from an economic point of view, the prediction of currency volume issued has become an important research topic [1-3].

In contrast to the error correction model (ECM) for predicting the volume of currency issued [4-7], this study applies two types of forecasting techniques to predict the volume of currency issued in Taiwan. The first technique is the singlestage forecasting modeling method, which includes the autoregressive integrated moving average (ARIMA), the multiple regression (MR), and the artificial neural networks (ANNs). ARIMA is a suitable modeling technique for making currency predictions because seasonal effects will be significantly involved $[8,9]$. The ARIMA models are wellknown and flexible because they can represent different types of time series, such as pure autoregressive (AR), pure moving average (MA) and the combined AR and MA (ARMA) series. In addition, ARIMA models are widely used in forecasting many practical phenomena, such as product sales, food prices, and stock prices, among others [10-15]. However, the assumptions inherent to the linear form of ARIMA models often present difficulties in capturing the nonlinear pattern of the real data $[10,16,17]$. The MR model is also a widely used forecasting technique for many practical applications $[15,18-$ 20]. This model has often been used to forecast based on known marketing variables and macroeconomic measures [21]. However, MR modeling has been criticized for its strong modeling assumptions, such as variation homogeneity, and thus, its application has been limited. In contrast, the ANN modeling is data driven because it contains fewer a priori assumptions. Accordingly, the ANN is another alternative modeling scheme for predicting currency because the ANN allows to model nonlinearity and provides good forecasting characteristics $[16,17,22,23]$. As a result, the ANN has been reported to exhibit better forecasting capability than the regression technique [22-28]. However, ANN is criticized for 
its long training process in designing the optimal network topology and because it is difficult to identify the relative importance of potential input variables $[25,26,29,30]$.

The second proposed technique is the two-stage hybrid modeling scheme. The general concept of using a hybrid scheme is to capture different patterns in the data by taking advantage of each individual model's capability. The research findings indicated that the hybrid modeling is superior for improving the performance of each individual model $[13,16$, 31-36]. Since both MR and ANN are very suitable for modeling the currency issued, this study considers a combination of MR and ANN as the proposed hybrid model. Because a large number of input variables for the ANN modeling may not be appropriate [35], the proposed MR technique is able to select a lower number of explanatory variables that have a greater importance. In the second stage of the hybrid modeling scheme, the important variables then serve as inputs for the proposed ANN prediction model.

This hybrid model is then used to analyze a real monthly dataset containing one response variable (i.e., Taiwan's currency) and twenty associated explanatory variables obtained from January 1996 to December 2011. The real dataset makes it possible to compare predictions about Taiwan's currency using the single-stage models and two-stage hybrid models. This study applies the first 14 years of data to build the forecasting models and then performs a confirmation test using the last two years of data. The rest of the study is organized in the following manner. Various forecasting methodologies are discussed in Section 2. The development and design of the single- and two-stage hybrid models are presented in Section 3. Practical data regarding the volume of currency issued in Taiwan are used to verify the single-stage models and the proposed hybrid models. The final section discusses the research findings and conclusions inferred from this study.

\section{The Methodologies}

In this study, we employ single-stage forecasting techniques, ARIMA, MR, and ANN, as well as a two-stage hybrid technique, MR-ANN, to predict the volume of currency issued. Additionally, to compare the performance of the different performance models, a real Taiwanese currency dataset is analyzed. The dataset consists of 192 records. Each sample record consists of 20 variables that are summarized in Table 1 (i.e., please see http://www.cbc.gov.tw/mpl.html for more details and descriptions about the dataset).

2.1. ARIMA Modeling. The time series data can be simply defined as observations made in a sequential order. Because seasonal effects are involved in the prediction of the currency issued, time series forecasting techniques should be used. Box and Jenkins $[8,9]$ have developed a well-known approach, ARIMA, for the prediction of a time series data. The ARIMA technique has proven viable for a wide variety of applications, ranging from economics and finance to traffic control and engineering.
TABLE 1: Explanatory variable definitions in the dataset.

\begin{tabular}{ll}
\hline Variable & Meaning \\
\hline$Y$ & Issued currency \\
$X_{1}$ & Stock price index \\
$X_{2}$ & Total trading value on the stock market \\
$X_{3}$ & Total trading value on the bond market \\
$X_{4}$ & Total trade value on call loan \\
$X_{5}$ & Interbank closing spot exchange rate (NT\$/US\$) \\
$X_{6}$ & The price of gold \\
$X_{7}$ & Annual growth rate of consumer prices \\
$X_{8}$ & Annual turnover rate of checking accounts \\
$X_{9}$ & Number ratio of dishonored checks \\
$X_{10}$ & Foreign-exchange reserves \\
$X_{11}$ & Discount rate \\
$X_{12}$ & Interest rate of accommodations with collateral \\
$X_{13}$ & 1-month deposit rates \\
$X_{14}$ & 1-year deposit rates \\
$X_{15}$ & Interbank call loan market interest rate \\
$X_{16}$ & Cp rates \\
$X_{17}$ & Deposits of major financial institutions \\
$X_{18}$ & Amount outstanding of bond market \\
$X_{19}$ & Unemployment rate \\
$X_{20}$ & Month of lunar new year \\
\hline &
\end{tabular}

A general ARIMA model can be described as follows:

$$
\begin{aligned}
\phi_{p}(B) & \Phi_{P}\left(B^{L}\right)\left(1-B^{d}\right)\left(1-B^{L}\right)^{D} Z_{t} \\
= & \delta+\theta_{q}(B) \Theta_{Q}\left(B^{L}\right) a_{t},
\end{aligned}
$$

where $\delta$ is an unknown constant, $Z_{t}$ are the working series values as a function of time $t$, which are stationary after fitting a suitable transformation from the original time series $Y, d, D$ are the values of nonseasonal and seasonal transformations, respectively, $a_{t}$ is white noise at time $t$, which independent and identical (iid) with normal distribution, $p, P, q$ and $Q$ are the order (parameters) of autoregressive (AR) and moving average (MA) models, respectively, $B$ is the backward shift operator, defined as $B^{i} Z_{t}=Z_{t-i}$, and $L$ are the number of months in a year, and $L=12$ for the monthly data. $\phi_{p}(B)$ is a polynomial function for a nonseasonal AR model, defined as $\phi_{p}(B)=\left(1-\phi_{1} B-\phi_{2} B^{2}-\cdots-\phi_{p} B^{p}\right), \Phi_{P}\left(B^{L}\right)$ is a polynomial function for a seasonal AR model, defined as $\Phi_{P}\left(B^{L}\right)=\left(1-\Phi_{1, L} B-\Phi_{2, L} B^{2 L}-\cdots-\Phi_{P, L} B^{P L}\right), \theta_{q}(B)$ is a polynomial function for a nonseasonal MA model, defined as $\theta_{q}(B)=\left(1-\theta_{1} B-\theta_{2} B^{2}-\cdots-\theta_{q} B^{q}\right)$, and $\Theta_{Q}\left(B^{L}\right)$ is A polynomial function for a seasonal MA model, defined as $\Theta_{\mathrm{Q}}\left(B^{L}\right)=\left(1-\Theta_{1, L} B-\Theta_{2, L} B^{2 L}-\cdots-\Theta_{\mathrm{Q}, L} B^{\mathrm{Q}, L}\right)$.

Typically, the original time series (i.e., $Y_{t}$ ) values are transformed into a stationary working series (i.e., $Z_{t}$ ) to fit the ARIMA models. The transformation is usually performed using four combinations of $d$ and $D$; that is, $(d, D)=(0,0)$, $(d, D)=(1,0),(d, D)=(0,1)$, and $(d, D)=(1,1)$. Once the stationary working series have been obtained, we can apply the sample autocorrelation function (SAF) and sample 
partial autocorrelation function (SPAF) to determine the order of $p, P, q$, and $Q$ for the seasonal ARIMA models. After performing a diagnostic assessment for the parameters and residuals, we obtain the forecasting models. In this study, the prediction capability of the models is compared using three criteria, including mean absolute percentage error (MAPE), root mean square error (RMSE), and mean absolute difference (MAD). These prediction measurements are defined as follows:

$$
\begin{aligned}
\text { MAPE } & =\frac{1}{n} \sum_{t=1}^{n} \frac{\left|e_{t}\right|}{Y_{t}} \times 100, \\
\mathrm{RMSE} & =\sqrt{\frac{1}{n} \sum_{t=1}^{n} \mid\left(e_{t}\right)^{2}} \\
\mathrm{MAD} & =\frac{1}{n} \sum_{t=1}^{n}\left|e_{t}\right|,
\end{aligned}
$$

where $e_{t}$ stands for the value of the residual at time $t$.

2.2. ANN Modeling. In recent years, ANN has been widely applied in engineering, education, social science, medical research, business, and forecasting. A neural network is a massively parallel system comprised of highly interconnected, interacting processing elements based on neurobiological models [36]. Due to its associated memory characteristic and its generalization capability, ANN has been increasingly utilized for modeling nonstationary processes $[35,37-42]$.

ANN can be classified into two categories: feedforward and feedback networks [43]. The nodes in the ANN can be divided into three layers: the input, the output, and one or more hidden layers. The output of each neuron in the input layer is the same as the input to that neuron. For each neuron $j$ in the hidden layer and neuron $k$ in the output layer, the net inputs are given by

$$
\text { net }_{j}=\sum_{i} w_{j i} \times o_{i}, \quad \text { net }_{k}=\sum_{j} w_{k j} \times o_{j},
$$

where $i(j)$ is a neuron in the previous layer, $o_{i}\left(o_{j}\right)$ is the output of node $i(j)$ and $w_{j i}\left(w_{k j}\right)$ is the connection weight from neuron $i(j)$, to neuron $j(k)$. The neuron outputs are given by

$$
\begin{gathered}
o_{i}=\text { net }_{i}, \\
o_{j}=\frac{1}{1+\exp ^{-\left(\text {net }_{j}+\theta_{j}\right)}}=f_{j}\left(\text { net }_{j}, \theta_{j}\right), \\
o_{k}=\frac{1}{1+\exp ^{-\left(\text {net }_{k}+\theta_{k}\right)}}=f_{k}\left(\text { net }_{k}, \theta_{k}\right),
\end{gathered}
$$

where net ${ }_{j}\left(\right.$ net $\left._{k}\right)$ is the input signal from the external source to the node $j(k)$ in the input layer and $\theta_{j}\left(\theta_{k}\right)$ is the bias. The transformation function shown in (4) is called a sigmoid function. Because this is one of the most commonly utilized functions, it is applied in this study.

The generalized delta rule is the conventional technique used to derive the connection weights of the feedforward network [43]. Initially, a set of random numbers is assigned to the connection weights. Then, to determine the pattern $p$ with a target output vector $t_{p}=\left[t_{p 1}, t_{p 2}, \ldots, t_{p M}\right]^{T}$, the sum of the minimized squared error is given by

$$
E_{p}=\frac{1}{2} \sum_{j=1}^{M}\left(t_{p j}-o_{p j}\right)^{2}
$$

where $M$ is the number of output nodes.

2.3. Multiple Regression Modeling. Regression analysis is one of the most used statistical methods in modeling real-world applications. The modeling process involves setting up the relationships between one dependent (or response) variable and several independent (or explanatory) variables. The performance of the regression models is typically acceptable as long as the assumptions have been met. However, the assumptions of the regression model (e.g., variation homogeneity) often confine its application.

The general MR model can be represented as follows:

$$
y=\beta_{0}+\beta_{1} X_{1}+\beta_{2} X_{2}+\cdots+\beta_{p} X_{p}+\varepsilon,
$$

where $\beta_{0}, \beta_{1}, \beta_{2}, \ldots, \beta_{p}$ are referred to as model parameters and $\varepsilon$ is a random variable called the error term. The error term accounts for the variability in $y$ that cannot be explained by the linear effect of the $p$ explanatory variables. In general, there are four assumptions about the error term in the MR model, including the following:

(1) the $\varepsilon$ is a normally distributed random variable;

(2) the $\varepsilon$ is a random variable with a mean value of zero; that is, $E(\varepsilon)=0$;

(3) the variance of $\varepsilon$ is denoted by $\sigma^{2}$ and is the same for all values of the explanatory variables $X_{1}, X_{2}, \ldots, X_{p}$;

(4) the values of $\varepsilon$ are independent.

Because collinearity among independent variables will lead to imprecise estimates and serious stability problems, the collinearity diagnosis procedure should be performed first before screening significant independent variables. Some well-known criteria such as the variance inflation factor (VIF) or tolerance can be applied to examine collinearity. The VIF is defined as follows:

$$
\operatorname{VIF}\left(X_{j}\right)=\frac{1}{1-R_{j}^{2}}, \quad j=1,2, \ldots, k,
$$

where $R_{j}^{2}$ is the coefficient of determination of a regression $X_{j}$ that evaluates all other independent variables. The tolerance is defined as the reciprocal of the VIF. It has been suggested that when the value of VIF is greater than 10 , the sample set may have enough variation to suggest serious multicollinearity. Although several methodologies can be used to overcome the problems of collinearity, this study used the method in which one or several explanatory variables could be dropped from the model in order to lessen the collinearity and thus reduce the standard errors of the estimated regression coefficients of the explanatory variables remaining in the model. In addition 
TABLE 2: Parameter estimates for the time series model.

\begin{tabular}{|c|c|c|c|c|c|}
\hline \multicolumn{6}{|c|}{$\begin{array}{l}\text { The ARIMA procedure } \\
\text { Conditional least squares estimation }\end{array}$} \\
\hline Parameter & Estimate & Standard error & $t$ value & Approx. $\operatorname{Pr}>|t|$ & Lag \\
\hline MA1,1 & 0.59010 & 0.06694 & 8.82 & $<.0001$ & 1 \\
\hline AR1,2 & -0.83540 & 0.07871 & -10.61 & $<.0001$ & 12 \\
\hline AR1,3 & -0.60107 & 0.08684 & -6.92 & $<.0001$ & 24 \\
\hline
\end{tabular}

TABLE 3: Autocorrelation test for residuals for the ARIMA model.

\begin{tabular}{lccccccccc}
\hline To lag & Chi-square & DF & Pr $>$ ChiSq & \multicolumn{5}{c}{ Autocorrelations } \\
\hline 6 & 1.19 & 2 & 0.5512 & -0.048 & 0.039 & 0.022 & 0.049 & 0.019 & 0.018 \\
12 & 6.30 & 8 & 0.6141 & 0.046 & -0.010 & 0.019 & 0.086 & 0.092 & 0.108 \\
18 & 14.87 & 14 & 0.3871 & 0.216 & 0.028 & 0.021 & 0.045 & 0.002 & 0.006 \\
24 & 22.11 & 20 & 0.3344 & 0.028 & -0.005 & -0.024 & 0.006 & 0.194 & -0.018 \\
30 & 23.62 & 26 & 0.5979 & 0.086 & 0.001 & 0.012 & -0.014 & -0.015 & -0.002 \\
36 & 24.85 & 32 & 0.8121 & -0.020 & -0.020 & -0.015 & -0.063 & 0.029 & 0.017 \\
\hline
\end{tabular}

TABLE 4: The topology setting results for ANN model alone.

\begin{tabular}{lc}
\hline & ANN alone \\
ANN topology & MAPE \\
\hline$\{20-18-1\}$ & 6.634 \\
$\{20-19-1\}$ & 5.876 \\
$\{20-20-1\}$ & 5.534 \\
$\{20-21-1\}$ & 5.940 \\
$\{20-22-1\}$ & 6.833 \\
\hline
\end{tabular}

to the simplicity and effectiveness, this method has another advantage of reducing the numbers of explanatory variables. This feature is quite suitable for hybrid modeling since it typically captures less explanatory variables for the initial stage of modeling.

In addition, when a large number of explanatory variables are involved in the MR design, a great amount of computation is required for examining a large volume of computer outputs, most of which is associated with poor MR models. As a consequence, three variable selection procedures are employed in this study. Those three selections include forward selection, backward elimination, and stepwise regression procedures. Given a dataset with twenty explanatory variables, this study uses those three selection procedures to determine the explanatory variables that lead to the best model. The selection procedures are iterative, wherein a single explanatory variable is added or deleted at each step of the procedure, and the new model is evaluated. The criterion for selecting an explanatory variable is based on $F$ statistics. The significant selected explanatory variables then serve as inputs to the ANN for the development of a two-stage, MR-ANN, hybrid model.

\section{Modeling Results and Analysis}

After performing various modeling techniques to predict the Taiwan's currency, the results are reported and discussed in this section.

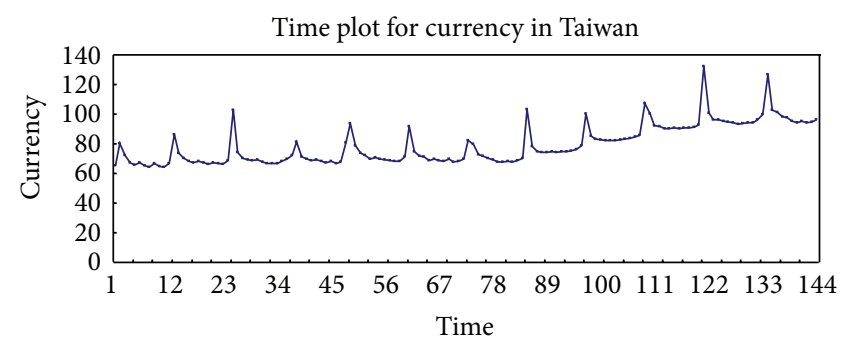

FIgURE 1: Time plot for currency issued in Taiwan (unit: $10^{8}$ NT\$).

3.1. ARIMA Modeling Results. For the ARIMA modeling, this study divides the currency data into two groups. The first group contains 168 samples used for the design of the model, and the second group contains 24 samples used for the confirmation of the model. Figure 1 displays the original time plot for the 144 currency observations. The time series of these 144 observations is not stationary, and a different transformation must be performed.

After performing the identification, estimation and diagnostic assessment steps using the SAS package, we obtain the parameter estimates provided in Table 2. Accordingly, we use the following ARIMA model (i.e., (8)) to predict the currency issued in Taiwan:

$$
y_{t}=-0.83540 y_{t-12}-0.60107 y_{t-24}+a_{t}-0.59010 a_{t-1} .
$$

Additionally, Table 3 provides the autocorrelation test for residuals for the ARIMA model. It indicates that the ARIMA model in (8) is adequate. records.

3.2. ANN Modeling Results. The purpose of using ANN is to predict the currency issued in Taiwan. The structure of thez ANN is described as follows. It has been reported that more than $75 \%$ of neural networks applications use the backpropagation neural network (BPNN) structure. Thus, this study uses the BPNN in designing the ANN forecasting model [3740]. For the ANN developed herein, this study utilizes 20 


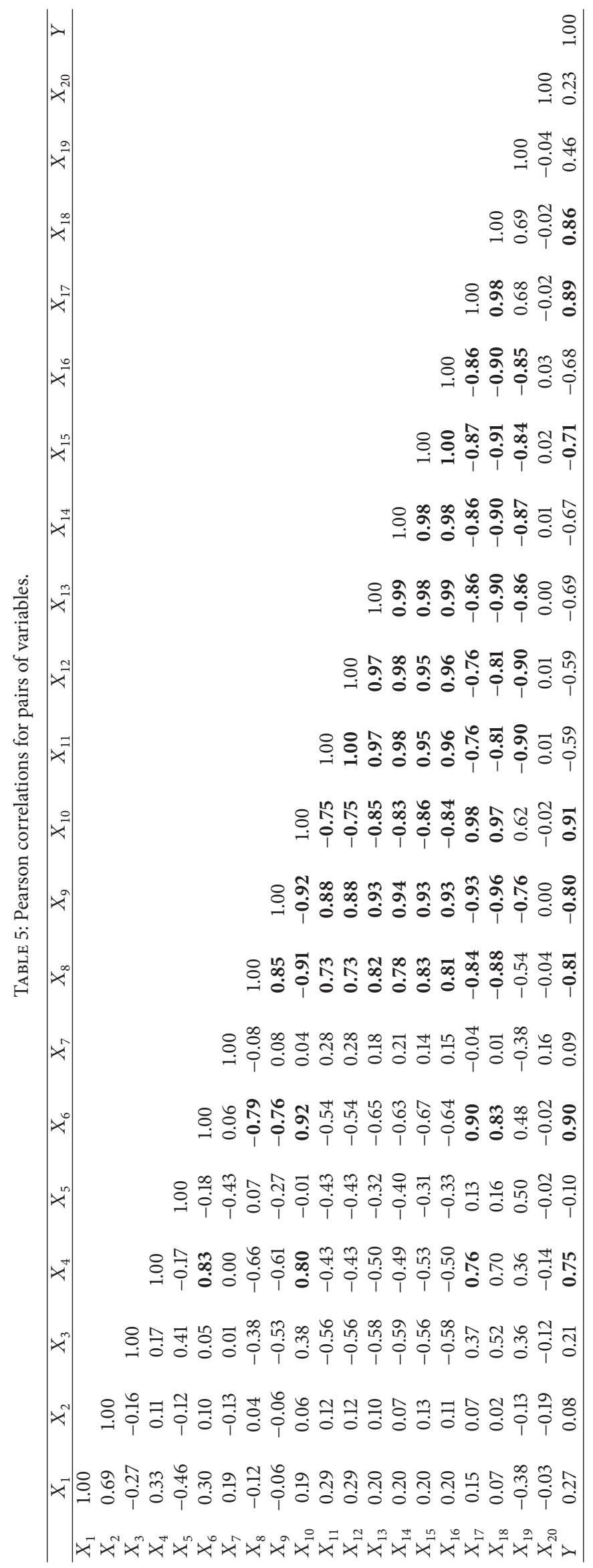


input nodes and one output node. The hidden nodes were set to $n \pm 2$, where $n$ is the number of input variables. Thus, the following hidden nodes are chosen: 18, 19, 20, 21 and 22. In this study, the training data and testing datasets include 168 and 24 data vectors for every possible parameter setting. Since the learning rate of 0.01 is a very effective setting $[35,40]$, this study sets the values of the learning setting as 0.01 for the ANN modeling. In addition, since MAPE is one of the most important performance measurements for the forecasting capability, this study uses the smallest MAPE as the criterion for selecting the ANN topology.

After performing the ANN modeling, we found that the $\{20-20-1\}$ topology with a learning rate of 0.01 provides the best results and a minimum testing MAPE. Here, $\left\{n_{\mathrm{i}}-n_{\mathrm{h}}-n_{o}\right\}$ stands for the number of neurons in the input layer, the number of neurons in the hidden layer, and the number of neurons in the output layer, respectively. Table 4 lists the corresponding MAPE values for various settings of the ANN topologies. Accordingly, the ANN topology of $\{20-20-1\}$ with a learning rate of 0.01 is chosen for the model of ANN alone.

3.3. MR Modeling Results. This study considers currency issued (i.e., $Y$ ) as the dependent variable and the twenty related economic variables (i.e., $X_{1}$ to $X_{20}$ ) as the explanatory variables. To exclude variables with high collinearity, the Pearson correlation coefficients between variables are used, and the results are provided in Table 5 . When the correlation coefficient $\rho_{X_{i}, X_{j}}$ between variables $X_{i}$ and $X_{j}$ is greater than 0.7 , we exclude the variable that has a lower relationship with $Y$ (i.e., exclude the variable with a smaller correlation coefficient $\left.\rho_{X, Y}\right)$. After discarding variables with high collinearity, seven explanatory variables, $X_{1}, X_{2}, X_{3}, X_{5}, X_{7}, X_{10}$, and $X_{20}$, remain. The associated VIF result is provided in Table 6 . Additionally, this model is simply referred to as the $\mathrm{MR}_{\mathrm{VIF}}$, and it is described as follows:

$$
\begin{gathered}
\text { Model_1 (with VIF checking) : } \mathrm{MR}_{\mathrm{VIF}} \\
Y=583375011.42+6158.38 X_{1}+476.78 X_{2} \\
-239.34 X_{3}-4071362.58 X_{5} \\
-1672867.30 X_{7}+1897.76 X_{10}+182059933.71 X_{20} .
\end{gathered}
$$

After using VIF to perform the parameter selection, this study used the typical statistical hypothesis tests to obtain the significant variables in the model. Accordingly, this study deleted the variables from seven retained variables (i.e., $X_{1}$, $X_{2}, X_{3}, X_{5}, X_{7}, X_{10}$, and $X_{20}$ ) whose absolute $t$ value is less than 1.96. In here, the type I error, $\alpha$, is chosen as 0.05 . This study utilized the SPSS with a testing sample, and the estimates of the parameters are provided in Table 6 . After performing the diagnostic assessment, we obtained an MR model that is selected by using the significance test. This model is referred to as the $\mathrm{MR}_{\mathrm{SIG}}$, and it is described in (10).

After using VIF to perform the parameter selection, this study used the typical statistical hypothesis tests to obtain the significant variables in the model. Accordingly, this study deleted the variables from seven retained variables (i.e., $X_{1}$,
TABLE 6: The results of parameter selection using VIF.

\begin{tabular}{lcc}
\hline & Coefficient estimates & VIF \\
\hline Constant & 583375011.42 & \\
$X_{1}$ & 6158.38 & 3.101 \\
$X_{2}$ & 476.78 & 2.477 \\
$X_{3}$ & -239.34 & 1.675 \\
$X_{5}$ & -4071362.58 & 1.899 \\
$X_{7}$ & -1672867.30 & 1.458 \\
$X_{10}$ & 1897.76 & 1.349 \\
$X_{20}$ & 182059933.71 & 1.111 \\
\hline
\end{tabular}

TABLE 7: The results of parameter estimates using the significance tests.

\begin{tabular}{lccc}
\hline & Coefficient estimates & $t$ value & Significance \\
\hline Constant & 515251473.83 & 44.42828 & 0.000 \\
$X_{3}$ & -258.41 & -3.2937 & 0.001 \\
$X_{10}$ & 1819.38 & 24.76641 & 0.000 \\
$X_{20}$ & 188422296.76 & 10.80766 & 0.000 \\
\hline
\end{tabular}

TABLE 8: The results of parameter estimates using the three selection procedures.

\begin{tabular}{lccc}
\hline & Coefficient estimates & $t$ value & Significance \\
\hline Constant & 450609032.27 & 17.169 & 0.000 \\
$X_{1}$ & 9385.75 & 2.733 & 0.007 \\
$X_{3}$ & -192.95 & -2.394 & 0.018 \\
$X_{10}$ & 1786.46 & 24.453 & 0.000 \\
$X_{20}$ & 191890298.23 & 11.191 & 0.000 \\
\hline
\end{tabular}

$X_{2}, X_{3}, X_{5}, X_{7}, X_{10}$, and $\left.X_{20}\right)$ whose absolute $t$ value is less than 1.96. In here, the type I error, $\alpha$, is chosen as 0.05 . This study utilized the SPSS with a testing sample, and the estimates of the parameters are provided in Table 7. After performing the diagnostic assessment, we obtained an MR model that is selected by using the significance test. This model is referred to as the $\mathrm{MR}_{\mathrm{SIG}}$, and it is described as follows:

$$
\begin{aligned}
& \text { Model_2(with significance tests) }: \mathrm{MR}_{\mathrm{SIG}} \\
& Y=515251473.83-258.41 X_{3}+1819.38 X_{10} \\
& +188422296.76 X_{20} .
\end{aligned}
$$

This study also used three selection techniques to develop the MR models for the currency issued. These three techniques include forward selection, backward elimination, and the stepwise regression analysis.

The concept of three variable selection procedures is described as follows. The forward selection is similar to the stepwise selection. The first explanatory variable selected for inclusion of the regression equation is the one with the largest positive or negative correlation with the dependent variable, $Y$. This explanatory variable is entered into the regression equation only if it satisfies the tolerance criterion for entry. If the first variable is entered, the explanatory variable which 
TABLE 9: The ANN topology setting for hybrid models.

\begin{tabular}{|c|c|c|c|c|c|}
\hline \multicolumn{2}{|c|}{$\mathrm{MR}_{\mathrm{VIF}}-\mathrm{ANN}$} & \multicolumn{2}{|c|}{$\mathrm{MR}_{\mathrm{SIG}}-\mathrm{ANN}$} & \multicolumn{2}{|c|}{$\mathrm{MR}_{\mathrm{SEL}}-\mathrm{ANN}$} \\
\hline ANN topology & MAPE & ANN topology & MAPE & ANN topology & MAPE \\
\hline$\{7-5-1\}$ & 4.272 & $\{3-1-1\}$ & 3.622 & $\{4-2-1\}$ & 4.135 \\
\hline$\{7-6-1\}$ & 3.576 & $\{3-2-1\}$ & 3.785 & $\{4-3-1\}$ & 3.801 \\
\hline$\{7-7-1\}$ & 3.603 & $\{3-3-1\}$ & 4.453 & $\{4-4-1\}$ & 3.659 \\
\hline$\{7-8-1\}$ & 4.073 & $\{3-4-1\}$ & 3.859 & $\{4-5-1\}$ & 3.526 \\
\hline$\{7-9-1\}$ & 3.530 & $\{3-5-1\}$ & 3.620 & $\{4-6-1\}$ & 3.672 \\
\hline
\end{tabular}

is not in the regression equation that has the largest partial correlation is considered next. The forward selection procedure would stop when there are no explanatory variables that meet the entry criterion. The back elimination procedure initially considers all explanatory variables to be included in the regression equation and then sequentially removed. The explanatory variable with the smallest partial correlation with the dependent variable is first for the removal. If that variable meets the tolerance criterion for elimination, it is removed. After the first variable is removed, the variable remaining in the regression equation with the smallest partial correlation is considered next. The procedure would stop when there are no variables in the regression equation that satisfy the removal criteria. At each step for the stepwise selection procedure, the explanatory variable which is not in the regression equation that has the smallest probability of $F$ is entered, if the probability is sufficiently small. Explanatory variables that have already existed in the regression equation are removed if their probability of $F$ is sufficiently large. The stepwise selection procedure would stop when no more variables are eligible for inclusion or removal.

In our experiment, all those three selection procedures resulted in the same MR model. Table 8 shows the SPSS results of the parameter estimates. This model is referred to as the $\mathrm{MR}_{\mathrm{SEL}}$, and it is described as follows:

$$
\begin{aligned}
& \text { Model_3 (with selection procedures) }: \mathrm{MR}_{\mathrm{SEL}} \\
& \begin{aligned}
Y= & 450609032.27+9385.75 X_{1}-192.95 X_{3} \\
& +1786.46 X_{10}+191890298.23 X_{20} .
\end{aligned}
\end{aligned}
$$

3.4. Hybrid Modeling Results. In our proposed two-step hybrid model, the first step is to obtain the appropriate input variables for the ANN model. Because this study utilizes different MR modeling selections, the explanatory variables in $\mathrm{MR}_{\mathrm{VIF}}, \mathrm{MR}_{\mathrm{SIG}}$, and $\mathrm{MR}_{\mathrm{SEL}}$ models serve as the input variables for ANN. Accordingly, this study employs three combinations of $\mathrm{MR}$ and $\mathrm{ANN}$ as the candidate hybrid models, wherein combinations of $\mathrm{MR}_{\mathrm{VIF}}$ and $\mathrm{ANN}, \mathrm{MR}_{\mathrm{SIG}}$ and $\mathrm{ANN}$, and $\mathrm{MR}_{\mathrm{SEL}}$ and $\mathrm{ANN}$ are referred to as $\mathrm{MR}_{\mathrm{VIF}^{-}}-\mathrm{ANN}, \mathrm{MR}_{\mathrm{SIG}^{-}}$ $A N N$, and $M_{S E L}-A N N$, respectively.

When the first stage of hybrid modeling is completed, the ANN topology settings are established. Table 9 displays the various ANN topology settings for the hybrid models. As a result, we found that the $\{7-9-1\},\{3-5-1\},\{4-5-1\}$ topologies with a learning rate of 0.01 provide the best result for $\mathrm{MR}_{\mathrm{VIF}^{-}}$ $\mathrm{ANN}, \mathrm{MR}_{\mathrm{SIG}}-\mathrm{ANN}$ and $\mathrm{MR}_{\mathrm{SEL}}-\mathrm{ANN}$, respectively.
3.5. The Modeling Results and Analysis. This study develops various forecasting models to predict the volume of currency issued in Taiwan. Table 10 provides the forecasting results, as well as the MAPE, MSE, and MAD of those models. A low MAPE, MSE, or MAD is associated with better forecasting accuracy. In comparison to the MAPE performance for the single-stage models in Table 10, we note that MR models exhibit better performance than the ARIMA and ANN models. However, the MSE, and MAD values of the MR models are larger than those of the ARIMA and ANN models. Accordingly, the forecasting results reveal that there is no significant difference among those single-stage models.

Nevertheless, our proposed hybrid models provide more accurate results than the single-stage models. In terms of MAPE, MSE or MAD, the three hybrid models are all lower than the four single-stage models. The MAPE percentage improvements of the proposed $\mathrm{MR}_{\mathrm{VIF}}-\mathrm{ANN}$ model over the four single stage models, ARIMA, $\mathrm{ANN}, \mathrm{MR}_{\mathrm{SIG}}$, and $\mathrm{MR}_{\mathrm{SEL}}$ for the 24-period forecasts are 36.52\%, 36.21\%, 17.73, and $15.39 \%$, respectively. Table 11 provides a comparison with respect to the overall improvement percentage in the singlestage models. As shown in Tables 10 and 11, the proposed hybrid models outperform all single-stage models.

\section{Conclusions}

The accurate prediction of currency volume issued is very important for the economic development of a country. This study performed a comparison of single-stage and hybrid models in predicting the volume of currency issued in Taiwan.

Because it is difficult to fully capture the characteristics of the real data, the hybrid scheme can be a good practical modeling approach. In this study, the concept of the proposed hybrid scheme takes advantage of each component model's unique capability to capture patterns in the currency data. Different combinations of hybrid technique were proposed to overcome the deficiencies of single models and yield more accurate prediction results. In this study, the MAPE, MSE, and MAD are used to measure the forecasting capability. The forecasting results reveal that the hybrid models are more fruitful methods for improving the forecasting performance of each single-stage model.

The proposed hybrid technique is more effective than the single-stage modeling. However, due to the difficulty of obtaining other countries' datasets, we are unable to perform the same procedures in reference to other countries. We do 
TABLE 10: Performance comparison of hybrid and single-stage models.

\begin{tabular}{lccc}
\hline & MAPE & RMSE & MAD \\
\hline Single-stage models & & & \\
$\quad$ ARIMA & 5.561 & 90211779 & 70983810 \\
ANN & 5.534 & 99438863 & 71830752 \\
MR $_{\text {SIG }}$ & 4.291 & 662791312 & 395781618 \\
$\quad$ MR $_{\text {SEL }}$ & 4.172 & 666308675 & 398221374 \\
Proposed hybrid models & & & \\
MR $_{\text {VIF }}$-ANN & 3.530 & 84906894 & 47388848 \\
MR $_{\text {SIG }}$-ANN & 3.484 & 83240416 & 46661477 \\
MR $_{\text {SEL }}$-ANN & 3.459 & 83909091 & 46313136 \\
\hline
\end{tabular}

TABLE 11: Improvement of the proposed models in comparison with the single-stage models.

\begin{tabular}{lccc}
\hline Models & MAPE (\%) & RMSE (\%) & MAD (\%) \\
\hline $\begin{array}{l}\text { Proposed hybrid } \\
\text { MR }_{\text {VIF }} \text {-ANN model }\end{array}$ & & & \\
ARIMA & 36.52 & 5.88 & 33.24 \\
ANN & 36.21 & 14.61 & 34.03 \\
MR $_{\text {SIG }}$ & 17.73 & 87.19 & 88.03 \\
MR $_{\text {SEL }}$ & 15.39 & 87.26 & 88.10 \\
Proposed hybrid & & & \\
MR $_{\text {SIG }}$-ANN model & & & \\
ARIMA & 37.35 & 7.73 & 34.26 \\
ANN $_{\text {MR }}$ & 37.04 & 16.29 & 35.04 \\
MR $_{\text {SEL }}$ & 18.81 & 87.44 & 88.21 \\
Proposed hybrid $_{\text {MR }_{\text {SEL }} \text {-ANN model }}$ & 16.49 & 87.51 & 88.28 \\
ARIMA $_{\text {ANN }}$ & & & \\
MR $_{\text {SIG }}$ & 37.80 & 6.99 & 34.76 \\
MR $_{\text {SEL }}$ & 37.50 & 15.62 & 35.52 \\
\hline
\end{tabular}

believe that the proposed hybrid approach is suitable for forecasting the currency issued for other countries in addition to Taiwan. We have described a framework for integrating several frequently used MR modeling methods and ANN techniques. The extension of these two-stage hybrid procedures to other techniques is currently under investigation.

\section{Acknowledgment}

This research was supported in part by the National Science Council of Taiwan, Grant no. NSC 99-2221-E-030-014-MY3.

\section{References}

[1] D. Maltritz and S. Eichler, "Currency crisis prediction using ADR market data: an options-based approach," International Journal of Forecasting, vol. 26, no. 4, pp. 858-884, 2010.
[2] G. C. Arquette, W. O. Brown Jr., and R. C. K. Burdekin, "US ADR and Hong Kong H-share discounts of Shanghai-listed firms," Journal of Banking and Finance, vol. 32, no. 9, pp. 19161927, 2008.

[3] G. L. Kaminsky and C. M. Reinhart, “The twin crises: the causes of banking and balance-of-payments problems," American Economic Review, vol. 89, no. 3, pp. 473-500, 1999.

[4] D. F. Hendry and N. R. Ericsson, "An econometric analysis of UK money demand in monetary trends in the United States and United Kingdom by Milton Friedman and Anna J. Schwartz," American Economic Review, vol. 81, pp. 8-38, 1991.

[5] Y. Baba, D. F. Hendry, and R. M. Starr, "The demand for M1 in the U.S., 1960-1984," Review of Economics Studies, vol. 59, no. 1, pp. 25-61, 1992.

[6] D. F. Hendry, "Modeling the demand for narrow money in the United Kingdom and the United States. by David F. Hendry and Neil R. Ericsson," European Economic Review, vol. 35, no. 4, pp. 883-886, 1991.

[7] T. Yoshida, "On the stability of Japanese money demand function: estimation results using error the correction model," Monetary and Economic Studies, vol. 8, no. 1, pp. 1-48, 1990.

[8] G. E. P. Box and G. M. Jenkins, Time Series Analysis: Forecasting and Control, Holden-Day, San Francisco, NC, USA, 1970.

[9] G. E. P. Box, G. M. Jenkins, and G. C. Reinsel, Time Series Analysis: Forecasting and Control, John Wiley \& Sons, New Jersey, NJ, USA, 4th edition, 2008.

[10] P. F. Pai and C. S. Lin, "A hybrid ARIMA and support vector machines model in stock price forecasting," Omega, vol. 33, no. 6, pp. 497-505, 2005.

[11] Y. E. Shao, Y. F. Lin, and S. T. Yuan, "Integrated application of time series multiple-interventions analysis and knowledgebased reasoning," Journal of Applied Statistics, vol. 26, no. 6, pp. 755-766, 1999.

[12] Y. E. Shao, "Multiple intervention analysis with application to sales promotion data," Journal of Applied Statistics, vol. 24, no. 2, pp. 181-191, 1997.

[13] H. C. Co and R. Boosarawongse, "Forecasting Thailand's rice export: statistical techniques vs. artificial neural networks," Computers and Industrial Engineering, vol. 53, no. 4, pp. 610627, 2007.

[14] H. F. Zou, G. P. Xia, F. T. Yang, and H. Y. Wang, "An investigation and comparison of artificial neural network and time series models for Chinese food grain price forecasting," Neurocomputing, vol. 70, no. 16-18, pp. 2913-2923, 2007.

[15] V. R. Prybutok, J. Yi, and D. Mitchell, "Comparison of neural network models with ARIMA and regression models for prediction of Houston's daily maximum ozone concentrations," European Journal of Operational Research, vol. 122, no. 1, pp. 3140, 2000

[16] M. Khashei and M. Bijari, "An artificial neural network (p, d, q) model for timeseries forecasting," Expert Systems with Applications, vol. 37, no. 1, pp. 479-489, 2010.

[17] P. P. Zhang, "Time series forecasting using a hybrid ARIMA and neural network model," Neurocomputing, vol. 50, pp. 159-175, 2003.

[18] R. Sadownik and E. P. Barbosa, "Short-term Forecasting of industrial electricity consumption in Brazil," Journal of Forecasting, vol. 18, no. 3, pp. 215-224, 1999.

[19] C. W. Chu and G. P. Zhang, "A comparative study of linear and nonlinear models for aggregate retail sales forecasting," International Journal of Production Economics, vol. 86, no. 3, pp. 217231, 2003. 
[20] M. Khashei, A. Z. Hamadani, and M. Bijari, "A novel hybrid classification model of artificial neural networks and multiple linear regression models," Expert Systems with Applications, vol. 39, no. 3, pp. 2606-2620, 2012.

[21] A. Ustundag, "A hybrid model for forecasting sales in Turkish paint industry," International Journal of Computational Intelligence Systems, vol. 2, no. 3, pp. 277-287, 2009.

[22] A. M. S. Muniz, H. Liu, K. E. Lyons et al., "Comparison among probabilistic neural network, support vector machine and logistic regression for evaluating the effect of subthalamic stimulation in Parkinson disease on ground reaction force during gait," Journal of Biomechanics, vol. 43, no. 4, pp. 720-726, 2010.

[23] M. Caselli, L. Trizio, G. De Gennaro, and P. Ielpo, "A simple feedforward neural network for the PM10 forecasting: comparison with a radial basis function network and a multivariate linear regression model," Water, Air, and Soil Pollution, vol. 201, no. 1-4, pp. 365-377, 2009.

[24] V. S. Desai, J. N. Crook, and G. A. Overstreet Jr., "A comparison of neural networks and linear scoring models in the credit union environment," European Journal of Operational Research, vol. 95, no. 1, pp. 24-37, 1996.

[25] T. S. Lee, C. C. Chiu, C. J. Lu, and I. F. Chen, "Credit scoring using the hybrid neural discriminant technique," Expert Systems with Applications, vol. 23, no. 3, pp. 245-254, 2002.

[26] S. M. Chou, T. S. Lee, Y. E. Shao, and I. F. Chen, "Mining the breast cancer pattern using artificial neural networks and multivariate adaptive regression splines," Expert Systems with Applications, vol. 27, no. 1, pp. 133-142, 2004.

[27] A. Minbashian, J. E. H. Bright, and K. D. Bird, "A comparison of artificial neural networks and multiple regression in the context of research on personality and work performance," Organizational Research Methods, vol. 13, no. 3, pp. 540-561, 2010.

[28] K. Subramanian, V. M. Periasamy, M. Pushpavanam, and K. Ramasamy, "Predictive modeling of deposition rate in electrodeposition of copper-tin using regression and artificial neural network," Journal of Electroanalytical Chemistry, vol. 636, no. 12, pp. 30-35, 2009.

[29] H. M. Chung and P. Gray, "Special section: data mining," Journal of Management Information Systems, vol. 16, no. 1, p. 11, 1999.

[30] M. W. Craven and J. W. Shavlik, "Using neural networks for data mining," Future Generation Computer Systems, vol. 13, no. 2-3, pp. 211-229, 1997.

[31] Y. E. Shao, C. J. Lu, and Y. C. Wang, "A hybrid ICA-SVM approach for determining the quality variables at fault in a multivariate process," Mathematical Problems in Engineering, vol. 2012, Article ID 284910, 12 pages, 2012.

[32] G. P. Zhang, "A neural network ensemble method with jittered training data for time series forecasting," Information Sciences, vol. 177, no. 23, pp. 5329-5346, 2007.

[33] C. J. Lu, Y. E. Shao, and P. H. Li, "Mixture control chart patterns recognition using independent component analysis and support vector machine," Neurocomputing, vol. 74, no. 11, pp. 1908-1914, 2011.

[34] C. D. Hou and Y. E. Shao, "Integrated use of statistical-based approaches and machine learning techniques for tumors classification using microarray data," Journal of Biobased Materials and Bioenergy. In press.

[35] Y. E. Shao and H. D. Hou, "Change point determination for a multivariate process using a two-stage hybrid scheme," Applied Soft Computing, vol. 13, no. 3, pp. 1520-1527, 2013.
[36] C. J. Lu and Y. E. Shao, "Forecasting computer products sales by integrating ensemble empirical mode decomposition and extreme learning machine," Mathematical Problems in Engineering, vol. 2012, Article ID 831201, 15 pages, 2012.

[37] D. E. Rumelhart, D. E. Hinton, and R. J. Williams, Learning Internal Representations by Error Propagation in Parallel Distributed Processing, MIT Press Cambridge, Cambridge, Mass, USA, 1986

[38] C. C. Chiu, Y. E. Shao, T. S. Lee, and K. M. Lee, "Identification of process disturbance using SPC/EPC and neural networks," Journal of Intelligent Manufacturing, vol. 14, no. 3-4, pp. 379-388, 2003.

[39] Y. E. Shao and B. S. Hsu, "Determining the contributors for a multivariate SPC chart signal using artificial neural networks and support vector machine," International Journal of Innovative Computing, Information and Control, vol. 5, no. 12, pp. 48994906, 2009.

[40] Y. E. Shao, C. J. Lu, and C. C. Chiu, "A fault detection system for an autocorrelated process using SPC/EPC/ANN and SPC/EPC/ SVM schemes," International Journal of Innovative Computing, Information and Control, vol. 7, no. 9, pp. 5417-5428, 2011.

[41] A. Azadeh, M. Saberi, A. Kazem, V. Ebrahimipour, A. Nourmohammadzadeh, and Z. Saberirw, "A flexible algorithm for fault diagnosis in a centrifugal pump with corrupted data and noise based on ANN and support vector machine with hyperparameters optimization," Applied Soft Computing, vol. 13, no. 3, pp. 1478-1485, 2013.

[42] R. Hedjar, "Adaptive neural network model predictive control," International Journal of Innovative Computing, Information and Control, vol. 9, no. 3, pp. 1245-1257, 2013.

[43] B. Cheng and D. M. Titterington, "Neural networks: a review from a statistical perspective," Statistical Science, vol. 9, no. 1, pp. 2-30, 1994. 


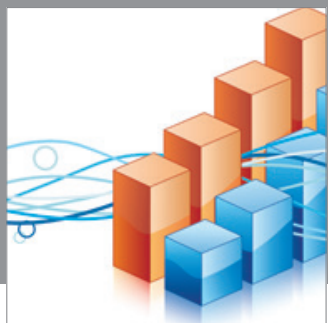

Advances in

Operations Research

mansans

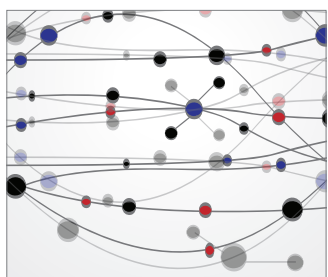

The Scientific World Journal
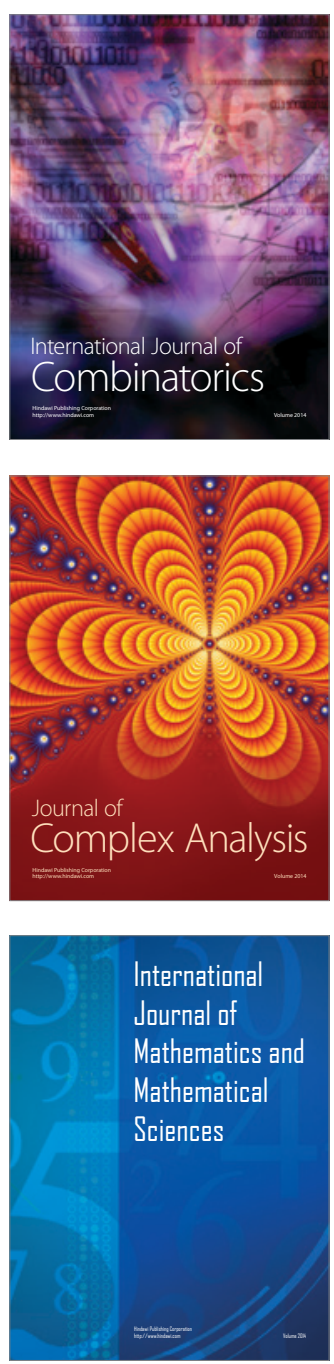
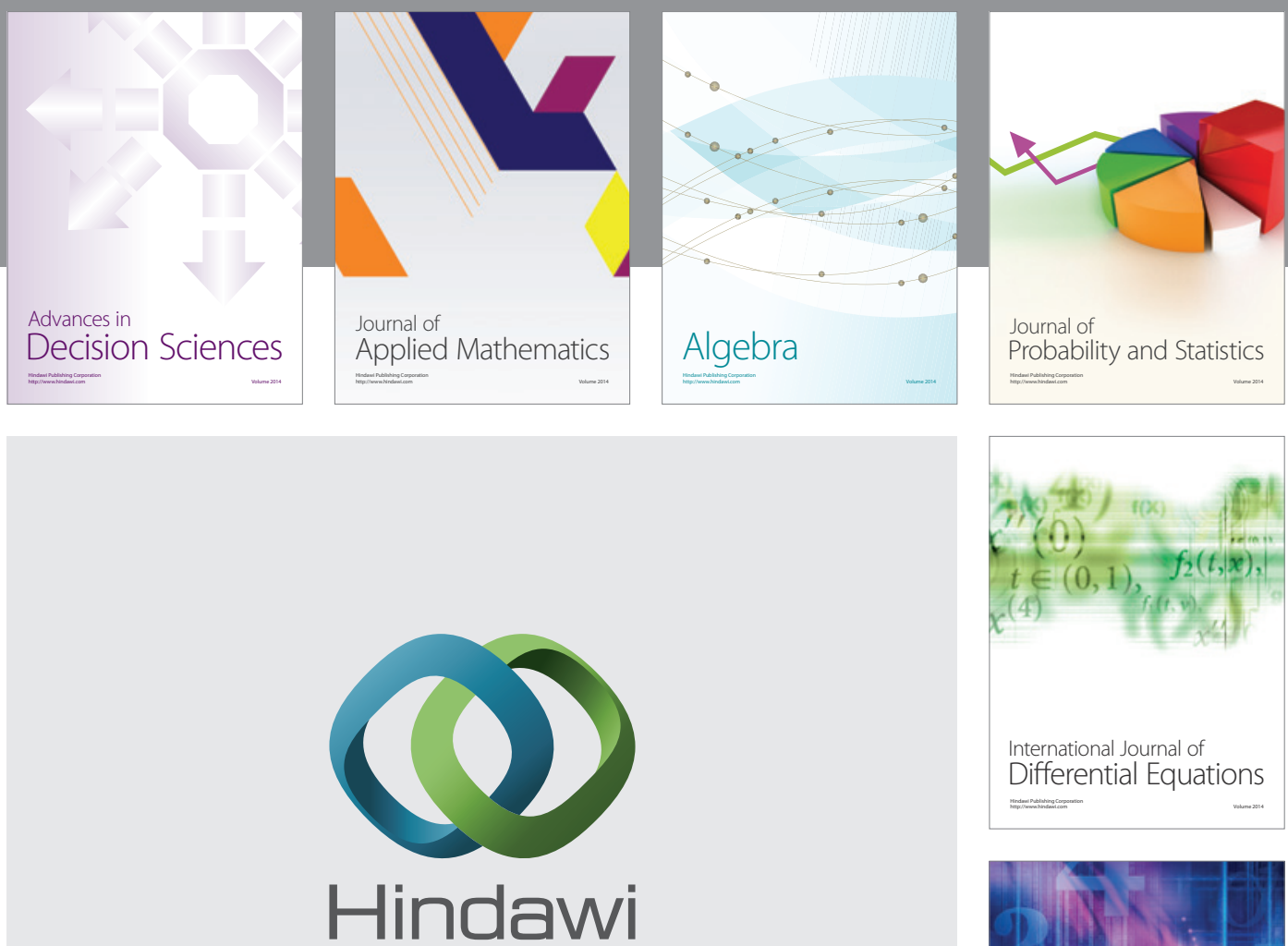

Submit your manuscripts at http://www.hindawi.com
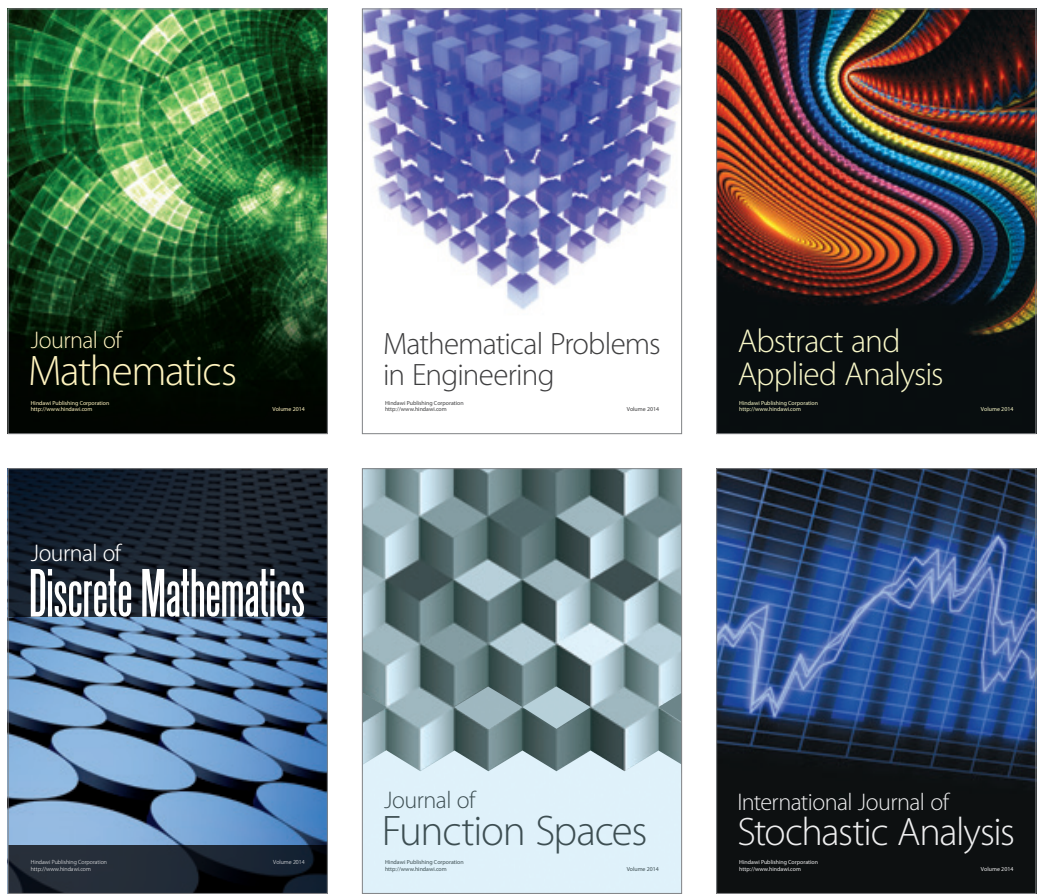

Journal of

Function Spaces

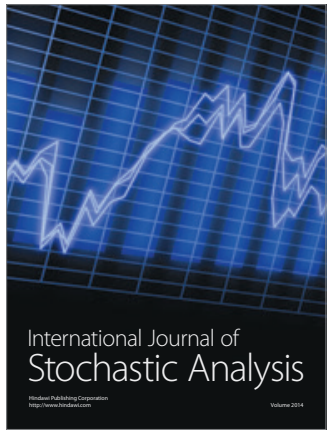

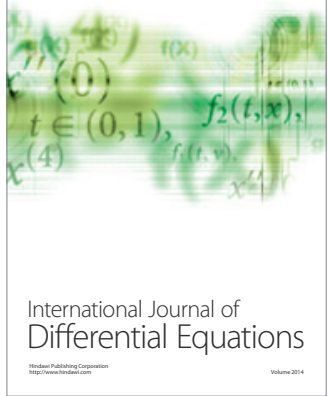
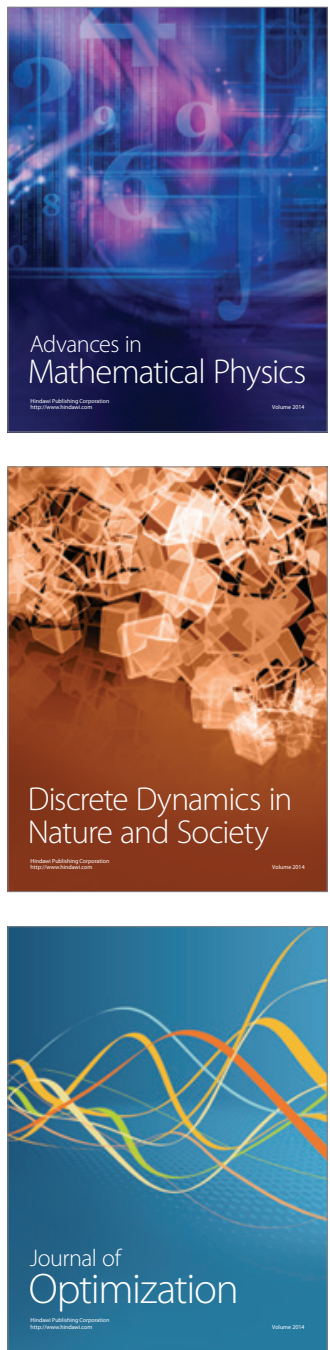